\title{
Upper Respiratory Symptoms Worsen over Time and Relate to Clinical Phenotype in COPD
}

Arturo Huerta ${ }^{1,3}$, MD, MSc, FCCP (ahuerta@clinic.ub.es), Gavin Donaldson², PhD (g.donaldson@imperial.ac.uk), Richa Singh, MD² (richa.singh@imperial.ac.uk), Alex Mackay, $\mathrm{MD}^{2}$ (alexander.mackay@ucl.ac.uk), James Allinson, $\mathrm{MD}^{2}$ (j.allinson@imperial.ac.uk), Simon Brill, $M D^{2}$ (s.brill@imperial.ac.uk), Beverly Kowlessar, RN² (b.kowlessar@imperial.ac.uk), Antoni Torres, MD, PhD ${ }^{3}$ (atorres@clinic.ub.es), Jadwiga A Wedzicha, MD, PhD²

(j.wedzicha@imperial.ac.uk)

\section{Author Affiliations:}

${ }^{1}$ Emergency Department, Medicine Section. Institut $d^{\prime}$ Investigacions Biomèdiques August Pi i Sunyer. Hospital Clinic de Barcelona. Barcelona, Spain. CibeRES 06/06/0028

${ }^{2}$ Airways Disease Section. Imperial College London. Royal Brompton Hospital. London, UK

${ }^{3}$ Institut Clínic del Tòrax, Servei de Pneumologia. Hospital Clinic de Barcelona. CibeRES 06/06/0028. Barcelona, Spain

\section{Corresponding Author:}

Arturo Huerta García, MD, MSc, FCCP

Emergency Department - Medicine Section

Institut d'Investigacions Biomèdiques August $\mathrm{Pi}$ i Sunyer.

Hospital Clinic de Barcelona. CIBeRES 06/06/0028

C. Villarroel 170 Esc. 4 Planta 2

Barcelona, Spain 08036

e-mail: ahuerta@clinic.ub.es

Tel: +34 932.275.400 ext 9384/ Fax: +34 932.275.549

Author Contributions: JAW, AH (principal contributors and guarantors) - overall study coordinators, study conception, results interpretation, manuscript write-up and review. GDstudy conception, data analysis, results interpretation, manuscript write-up and review. ATstudy conception, results interpretation, manuscript write-up and review. RS, AM, JA, SB, BK - Data acquisition, manuscript write-up and review.

Funding: AH was supported by generous grants from: the European Respiratory Society (ERS Short Term Fellowship STRTF 2012-204), la Escola de Doctorat de la Facultat de Medicina de la Universitat de Barcelona (UB) - Ajuts de mobilitat internacional, the Catalan Society of Pulmonology - SOCAP (Ajut d'estades al estranger 2012), the Spanish Society of Pulmonology (SEPAR) and FUCAP (Fundacio Catalana de Pneumologia).

Keywords: COPD, Upper respiratory symptoms, Phenotype, CAT score

Total Word Count: 2841 


\section{Abstract}

Rationale: How nasal symptoms in patients with COPD change over time and resolve during natural occurring exacerbation has never been described.

Methods: Patients in the London COPD cohort were asked about the presence of nasal symptoms (nasal discharge, sneezing, post-nasal drip (PND), blocked nose and anosmia) over an 8-year period (2005-2013) every three months at routine clinic visits at stable state and daily during exacerbations with the use of diary cards. Data was prospectively collected and in a subgroup of patients COPD Assessment Test (CAT) and human rhinovirus (HRV) identification by PCR was available. Patients were also defined as infrequent/frequent exacerbators ( $<2$ or $\geq 2$ exacerbations/year).

Measurements and Main Results: On 4368 visits, 209 patients with COPD were asked about their nasal symptoms. On 2033 visits, when the patients were stable, the odds ratio (OR) for nasal discharge increased by $1.32 \%$ per year ( $95 \% \mathrm{Cl} 1.19-1.45 ; \mathrm{p}<0.001)$; sneezing $1.16 \%$ (1.05-1.29; $p=0.005)$; post-nasal drip 1.18\% (1.03-1.36; $p=0.016)$ and anosmia 1.19\% (1.031.37; $p=0.015)$. At exacerbation, nasal discharge was present for 7-daysand blocked nose, sneezing and post-nasal drip increased for just 3 days; anosmia did not change. Nasal discharge was more likely in frequent exacerbators; OR $1.96(1.17-3.28 ; p=0.011)$ and when present, COPD Assessment Test scores were higher by 1.06 units $(0.32-1.80 ; p=0.005)$ when stable and 1.30 units $(0.05$ to $2.57 ; p=0.042)$ at exacerbation.

Conclusion: Upper airway symptoms increase over time in COPD patients and are related to the frequent exacerbator phenotype. These longitudinal changes may be due to increasing airway inflammation or the disease progression. 
Chronic obstructive pulmonary disease (COPD) is a major cause of morbidity and mortality ${ }^{1}$.The natural history of COPD is characterized by the repeated occurrence of episodes called exacerbations that are manifested by an inflammatory response, mediated predominantly by neutrophils that increase respiratory symptoms ${ }^{2,3}$.

It is widely known that there is an association between rhinitis and asthma since $90 \%$ of patients with asthma have rhinitis and almost $30 \%$ of patients with allergic rhinitis develop asthma ${ }^{4}$. In COPD, nasal symptoms are not considered important, and sometimes they are neglected, as the disease is thought to be primarily due to airflow limitation in the lung ${ }^{5}$. However, whenever a person inhales smoke from tobacco or biomass fuels, the smoke penetrates into the whole airway from nose to distant alveoli. We have previously described the presence of chronic nasal and respiratory symptoms in COPD patients ${ }^{6}$, the relationship of the upper airway to lower airway bacteria ${ }^{7}$, the impact of nasal symptoms on quality of life ${ }^{8}$ and the correlation of systemic inflammation both in upper and lower airways during exacerbation ${ }^{9}$. The concept that COPD is a "pan-airway" disease in COPD is gaining support with different groups publishing data that strengthen the epidemiological evidence $^{10,11}$.

However, there is no evidence that the prevalence or severity of nasal symptoms changes over time and forms part of the natural progression of the disease and neither the influence on health status, as assessed with the COPD Assessment Test (CAT) score. The concept of time-related worsening of nasal symptoms in mature population have been reported and shown that despite fairly low self-reported prevalence of these disorders in large population studies, when validated, there is a high prevalence of hyposmia and anosmia in certain groups, especially the elderly ${ }^{12}$. 
Some explanations regarding toxic exposure, head trauma, autoimmunity, or aging itself can contribute to smell impairment, with different implications concerning prognosis and possible treatment. Often, the ENT specialists are the one that identify in first place these symptoms in patients with chronic rhinosinusitis.

Thus, the aim of this study was to evaluate the evolution and impact of upper airway symptoms in a well-defined COPD cohort when stable and at exacerbation.

\section{Methods}

\section{Study Subjects and Protocol}

A total of 209 patients enrolled in the London COPD Cohort were included between March 2005 and June 2013 (with at least one year of participation in the cohort). A complete flowchart on the visits and collected data is shown in Figure 1. The post-bronchodilator forced expiratory volume in 1 second $\left(\mathrm{FEV}_{1}\right)$ of all patients was $\leq 80 \%$ predicted from age, height, and sex and a FEV 1 /Forced Vital Capacity (FVC) ratio $<0.7^{5}$. A history of smoking, including smoking status and medical history were recorded. Data on nasal steroid sprays were collected from May 2010 onwards.

\section{Definition of Exacerbation and Monitoring}

Patients were also asked to record on a daily diary card any increase or occurrence in their lower airway symptoms. Dyspnoea, sputum purulence and sputum volume were defined as major symptoms and nasal discharge/congestion, wheeze, sore throat and cough were defined as minor symptoms. Exacerbations were defined as previously used by the London 
COPD Cohort ${ }^{13}$, as any change in one major symptom with at least one other (major or minor) for two consecutive days.

The annual exacerbation frequency was calculated for each patient using the number of days the patients had completed their diary between January 2005 and June 2013. Patients were then defined as infrequent exacerbators ( $<2$ exacerbations/year) or frequent group ( $\geq 2 /$ year) ${ }^{14}$.

\section{Nasal Symptoms}

All subjects were asked about the presence of nasal symptoms (runny nose/nasal discharge, sneezing, post-nasal drip (PND), blocked nose or anosmia /impaired smell) in the threemonths prior to their quarterly stable clinic review. From March 2011 - June 2013 (Figure 1) patients were asked to grade the severity of their nasal symptoms $(0=$ no symptom, $1=$ very mild, $2=$ mild, $3=$ moderate, $4=$ severe and $5=$ bad as can be). At exacerbation and during recovery they were asked if there was a presence of these five nasal symptoms on each day. For the purpose of this study, runny nose and nasal discharge are considered equivalent terms.

\section{Quality of Life Assessment Using the CAT Score}

In a subgroup of patients (Figure 1), during the stable baseline state and during exacerbations, patients were asked to complete the CAT at least once under supervision in clinic and once at home. The stable state was defined when more than 35 days after and 21 days before exacerbation onset. If the baseline CAT scores before exacerbation onset were unavailable, the score during post-exacerbation stability was used. 


\section{Sputum Collection and Processing}

At a number of the clinic visits (Figure 1), spontaneously expectorated sputum was collected. Quantitative Polymerase Chain Reaction (qPCR) was used to detect human rhinovirus using the ABI Prism 7500 Real-time qPCR System (Applied Biosystems) and validated primers ${ }^{15}$

\section{Statistical Analysis}

For the statistical analyses, Stata 13 (Stata Corp, Texas, USA) was used and data were expressed as percentages, mean \pm SEM or median \pm inter-quartile range. Comparisons between independent groups were made by chi-squared test, Student's $t$-test or Wilcoxon sign-rank test respectively. The correlations between the means of independent groups were evaluated with Pearson correlation test. A p-value $<0.05$ was considered statistically significant.

Trends over time were first assessed in a univariate analysis with random-effect logistic regression models that allowed for repeated measures in the same individual. These trends were then assessed into a multi-variate model that included smoking status and exacerbation frequency phenotype and their interaction with time. To assess whether nasal symptoms at exacerbation rose faster overtime compared to baseline nasal symptom, we also included in the multivariate model a term describing whether the data was recorded at baseline or exacerbation and its interaction with time. Results are reported as odds ratio for a 1-year interval with $95 \%$ confidence interval.

An analysis of variance was used to compare the severity of symptom over various time periods during the onset and recovery of exacerbation with data recorded at baseline. 
Random effects models that adjusted for repeated measures were used also for the analysis of the CAT score and its impact on nasal symptoms.

The Ethical Committee of the Royal Free Hospital granted approval for this study and all patients gave informed written consent.

\section{Results}

\section{Demography}

Table 1 shows the main characteristics of the 209 COPD patients included. The mean FEV 1 was $1.22 \mathrm{~L}(0.46) ; \mathrm{FEV}_{1}$ as \% predicted $50.1 \%$ (SD 16.4), age was 70.7 years (SD 8.7). Of the population, 130 were male (62\%) and 66 (32\%) were current smokers. A total of 13/140 (9.3\%) of patients on whom data was available, have been receiving nasal corticosteroids at some time. Due to the relation between bronchiectasis and sinusitis, none of the patients included for the purpose of this study had clinical findings of bronchiectasis (large volume of sputum or coarse crepitations) during clinical history or examination.

\section{Nasal Symptoms at Baseline and Changes over Time}

Data on nasal symptoms from 209 stable patients were recorded on 2033 clinic visits. Figure 2 shows that the overall prevalence of these symptoms increased over time. In a univariate analysis all the nasal symptoms increased over time in the overall population (all symptoms, all patients, $p<0.05)$, but in the multivariate analysis, the odds ratio (OR) for a runny nose/nasal discharge increased over time independently of exacerbation frequency or smoking status by $1.32 \%$ per year $(95 \% \mathrm{Cl} 1.19-1.45 ; p<0.001)$; sneezing $1.16 \%$ (1.05-1.29; 
$\mathrm{p}=0.005)$; post-nasal drip 1.18\% (1.03-1.36; $\mathrm{p}=0.016)$ and anosmia 1.19\% (1.03-1.37;

$p=0.015)$ but not blocked nose $O R=1.01 \%(0.90-1.14 ; p=0.811)$.

However, nasal discharge was more likely in frequent exacerbators; OR 1.96 (1.17-

3.28; $p=0.011)$. Current smokers were also more likely to experience impaired

smell/anosmia: OR $2.47(1.06-5.80 ; p=0.037)$.

\section{Time Course of Nasal Symptoms during Exacerbations}

Nasal symptoms were recorded during exacerbation between onset on day 0 and day 42 on 2197 visits; 838 visits were between 0 to +7 days of exacerbation onset. Of these 838 occasions, only the incidence of a nasal discharge and post-nasal drip increased over time, with OR $1.18(1.09-1.29 ; p<0.001)$ and $1.20(1.08-1.33 ; p<0.001)$ respectively, but these longitudinal changes were not significant after adjustment for smoking, frequent/infrequent exacerbator phenotype or interactions with time. The combination of the exacerbation data with the baseline data did not show any differences in the increased prevalence of nasal symptoms over time between the stable and exacerbation state.

\section{Time Course of Nasal Severity Score}

Figure 3 shows the severity of nasal symptoms at baseline, prodrome (days -7 to -1 ), onset (0-3 days) and during recovery from exacerbation in the overall population. In comparison with the baseline, nasal scores for runny nose/nasal discharge were higher on days 0-3 and days $4-7$ ( $p<0.001$ and $p<0.001$ respectively). Symptoms of a blocked nose, post-nasal drip and sneezing appeared to resolve faster than nasal discharge as scores where significantly higher on days $0-3(p=0.006, p=0.001$ and $p=0.005$ respectively) but by days $4-7$ were not significantly different from baseline $(p=0.693, p=0.069$ and $p=0.128$ respectively). The 
severity of anosmia did not change at exacerbation. The nasal severity scores were not higher than baseline on days during the prodrome (days -7 to -1 ) or during the later stages of exacerbation recovery, days 8-14, days $15-29$ or days 29 to 42 .

\section{CAT Score and Nasal Symptoms}

In a subgroup of patients (May 2011-June 2013) as stated in Figure 1, the CAT questionnaire was completed on 728 of the 2033 baseline visits and 347 of the 838 exacerbation visits in 0 to +7 days. Figure 4 shows that the CAT score were significantly different between baseline and at exacerbation in those with nasal discharge symptoms as it increased by 1.06 units $(0.32-1.80 ; p=0.005)$ and by $1.30(0.05$ to $2.57 ; p=0.042)$ respectively. CAT scores were higher by 3.6 units in frequent exacerbators but there was no difference in the effect of a runny nose on the CAT score between frequent and infrequent exacerbators $(p=0.062)$. The presence of any of the other nasal symptoms was not associated with differences in the CAT score.

\section{Associations of Nasal Symptoms with Human Rhinovirus (HRV)}

In a subgroup of patients, there were 311visits when qPCR on sputum samples collected was performed (Figure 1). HRV was detected only in 42/122 (34.4\%) sputum samples when symptoms of a blocked nose were present compared to $44 / 188$ when symptoms were absent (23.4\%; $p=0.034)$. Moreover42/122(34.4\%) positive samples coincided with sneezing but only $42 / 188(22.3 \% ; p=0.019)$ when this symptom was absent. With a nasal discharge, the percentages were $58.2 \%$ compared to $48.2 \%$ ( $p=0.083$ ); post nasal-drip $22.1 \%$ vs $21.7 \%$; $(p=0.927)$ and for anosmia $11.5 \%$ vs $9.0 \% ;(p=0.485)$. 


\section{Discussion}

This study for the first time shows that nasal symptoms in stable COPD patients increase progressively and shows their time-course during naturally occurring exacerbations. This study also relates the presence of nasal discharge to the frequent exacerbator phenotype. Moreover these data has been prospectively collected over a wide period of time (8 years) in a very well characterised cohort of COPD patients..

In a previous study by our group ${ }^{6}$, we found a prevalence of nasal symptoms of $75 \%$, with $52 \%$ of patients reporting nasal discharge and $45.9 \%$ reported sneezing. That study showed a trend towards increased nasal symptoms in frequent exacerbators compared to infrequent exacerbators, and that nasal symptoms were independent of smoking status. In the current study of 209 patients, we found that runny nose/nasal discharge was more common in frequent exacerbators, but none of the other symptoms were as strongly linked as this one to this phenotype. Nasal blockage and anosmia/impaired smell was more prevalent in current smokers.

Two clinical findings need to be emphasized in this study. First, the presence of nasal symptoms was different according to the frequent exacerbator phenotype, especially the presence of runny nose/nasal discharge. This may be in part due to impairment of mucociliary function in these patients and the increased mucosal inflammation in the upper airways as previously described ${ }^{16}$.Second, although some of the patients were former smokers, the nasal symptoms continued worsening over time in both frequent and infrequent exacerbators, strengthening the hypotheses that once prolonged smoke exposure arrives at a threshold, the inflammatory stimuli cannot easily be switched-off ${ }^{17}$. 
With experimental human models of exacerbations, it is known that the majority of patients experience worsening in both upper respiratory (sneezing, post-nasal drip, runny nose/nasal discharge) and lower symptoms ${ }^{18}$. Our study is the first to describe the time course during exacerbations typical of those seen in the community and triggered by viruses, bacteria or pollution. As expected at exacerbations in the overall population, nasal discharge was the most common symptom (frequent and infrequent patients) at the onset, together with post-nasal drip, with both symptoms worsening during the first seven days.

Post-nasal drip, blocked nose and sneezing as symptoms appeared in the early stages of exacerbation during the first three days, but these symptoms recovered faster. This clinical evolution of nasal symptoms at exacerbation was not related to the clinical phenotype or the current or past smoking exposure.

It is important to mention that not all-nasal symptoms recorded were related to the presence of the most common respiratory virus ( $\mathrm{HRV}$ as the cause of common cold) during exacerbations. Only nasal discharge and sneezing were consistently associated with HRV in all frequent/infrequent patients and these symptoms may be useful in identifying some exacerbations that may be early treated with anti-viral medications. This finding also agrees with studies concerning the transmission of respiratory viruses, since these agents were present in almost $50 \%$ of exacerbations ${ }^{19}$.

Anosmia/impaired smell did not change over the time of the exacerbation even at the onset when both lower and the other upper respiratory symptoms were at their worse. No differences in anosmia were found in regards to being a frequent or infrequent exacerbator patient. However, in general population, $70.5 \%$ of women and just $29.5 \%$ of men report a worsening of anosmia either during or post-upper respiratory tract infection ${ }^{20}$. 
It is possible that since our cohort is of $62 \%$ male COPD subjects, they were less likely to report a worsening of this symptom.

During the study a subgroup of patients completed the CAT questionnaire. This is a validated eight-item questionnaire designed to assess and quantify the impact of COPD symptoms on patient health status ${ }^{21}$ but it has been also shown to be a good tool in measuring severity ${ }^{22}$ and recovery from exacerbations ${ }^{23}$. It also has two principal advantages in that it can be used in different clinical settings (primary care and second care) and shows no variability between languages ${ }^{24}$.

Among all the nasal symptoms explored, only nasal discharge showed a rise in the CAT score, both in the stable state and during exacerbations as it was also the most prevalent symptom in our population during stable state and during exacerbations . Although it has not been properly established, a change of 1.6 units in the CAT score has been suggested as a threshold in the variation of the CAT score to arrive to the minimal clinically important difference ${ }^{24}$.In our study we showed a rise that might not be significant, but our study opens a new study area, regarding the impact of nasal symptoms on the CAT score in COPD.

A potential limitation of the study must be addressed, as we do not have sinus CTscans or ENT examination of all the patients (to assess the presence or absence of nasal polyps) but there is some evidence that the symptoms in both populations (with and without polyps) usually overlap ${ }^{25}$. We also do not have information on the possible presence of patients with the criteria for the asthma and COPD overlap syndrome as recently described by GINA-GOLD ${ }^{26}$.

Airway bacterial colonisation (BC) is common in latest phases of COPD and is well known that sputum samples correlate with bacteria isolated from lower airways by 
bronchoscopic samples ${ }^{27,28}$. The presence of bacteria increases local inflammation of the bronchi and elevation of systemic inflammatory biomarkers ${ }^{29}$. However, few studies have investigated bacterial colonisation of the upper airway in COPD patients ${ }^{7}$. Further studies in this field are needed to clarify the pathogenics of the sinonasal symptoms.

Some strains of the common microorganisms often associated with acute exacerbations (Non-typeable H. Influenzae, M. catarrhalis) can form biofilms. This could lead to biofilm-associated chronic rhinosinusitis. Although, several studies aimed at preventing biofilm formation with topical antimicrobials, surfactants, loop diuretics and macrolides have been tested ${ }^{30}$, none of these interventions have been studied in COPD patients.

A pilot study on 33 patients has recently been published on the use of nasal corticosteroids (fluticasone furoate) and showed that in patients assigned to the treatment group, there was a decrease of nasal IL-8 after 12 weeks of treatment and an amelioration of nasal symptoms ${ }^{31}$. However, little information is known on the frequent/infrequent phenotype in this paper or the response of eosinophilic inflammation (eotaxin response) in response to the use of nasal corticosteroids. A larger trial with more clinical characterisation in COPD patients is warranted.

In conclusion, we have shown that nasal symptoms are part of the natural evolution of COPD and that they worsen over time and are closely related to the frequent exacerbator phenotype. These symptoms should be taken into account when performing multidimensional evaluations of the patients, since they might also impact on assessment scores and health-related quality of life.

Acknowledgments: The authors want to acknowledge Siobhan George for her outstanding work doing the qPCR for rhinovirus in the cohort samples. 
Table 1. Demographics on population studied at the London COPD cohort

\begin{tabular}{|c|c|}
\hline & $\mathrm{N}=209$ \\
\hline Age (years) & $70.7(\mathrm{SD} 8.7)$ \\
\hline Gender (male) \% & $130(62 \%)$ \\
\hline Smoking (\%) & $66(31 \%)$ \\
Current smoker & $143(68 \%)$ \\
Former smoker & $106(50.7 \%)$ \\
\hline COPD & $75(35.9 \%)$ \\
Stage II & $28(13.4 \%)$ \\
\hline Stage III & $1.22(0.46)$ \\
\hline Stage IV & $50.1 \%(16.5)$ \\
\hline FEV $($ L) & $2.65(0.87)$ \\
\hline FEV F $_{1} \%$ predicted (SD) & $0.47(0.12)$ \\
\hline FVC (L) & $1.92(1-3.1)$ \\
\hline FEV1/FVC ratio & \\
\hline Number of exacerbations in the prior year & \\
\hline (median + interquartile range) & \\
\hline
\end{tabular}




\section{Figure Legends}

Figure 1. Flowchart of COPD patients included, data collected and samples processed during 8-years (2005-2013) in the London COPD cohort.

Figure 2. Percentage of patient-reported symptoms over time in the London COPD Cohort. Number refers to the number of visits where patients reported having nasal symptoms.

Figure 3. Time course of nasal symptoms during exacerbations in the London COPD Cohort graded and divided by clusters of days.

Figure 4. Effect of nasal discharge during stable state and at exacerbation and its impact on the CAT score.

Figure 5. Time course of nasal symptoms during exacerbations in patients with infrequent and frequent exacerbations. ${ }^{*} p<0.05$, chi-squared test. 


\section{References}

(1) Seemungal TA, Hurst JR, Wedzicha JA. Exacerbation rate, health status and mortality in COPD--a review of potential interventions. Int J Chron Obstruct Pulmon Dis 2009; 4:203223.

(2) Burge S, Wedzicha JA. COPD exacerbations: definitions and classifications. Eur Respir J Suppl 2003; 41:46s-53s.

(3) Mackay AJ, Hurst JR. COPD exacerbations: causes, prevention, and treatment. Med Clin North Am 2012; 96(4):789-809.

(4) Bachert C, Vignola AM, Gevaert P et al. Allergic rhinitis, rhinosinusitis, and asthma: one airway disease. Immunol Allergy Clin North Am 2004; 24(1):19-43.

(5) Global Strategy for Diagnosis, Management and Prevention of COPD. Available at: http://www.goldcopd.com/GuidelinesResources.asp (accessed 20 OCt 2013). 2013.

(6) Roberts NJ, Lloyd-Owen SJ, Rapado F et al. Relationship between chronic nasal and respiratory symptoms in patients with COPD. Respir Med 2003; 97(8):909-914.

(7) Hurst JR, Wilkinson TM, Perera WR et al. Relationships among bacteria, upper airway, lower airway, and systemic inflammation in COPD. Chest 2005; 127(4):1219-1226.

(8) Hurst JR, Wilkinson TM, Donaldson GC et al. Upper airway symptoms and quality of life in chronic obstructive pulmonary disease (COPD). Respir Med 2004; 98(8):767-770.

(9) Hurst JR, Perera WR, Wilkinson TM et al. Systemic and upper and lower airway inflammation at exacerbation of chronic obstructive pulmonary disease. Am J Respir Crit Care Med 2006; 173(1):71-78.

(10) Hens G, Vanaudenaerde BM, Bullens DM et al. Sinonasal pathology in nonallergic asthma and COPD: 'united airway disease' beyond the scope of allergy. Allergy 2008; 63(3):261-267.

(11) Halawi AM, Smith SS, Chandra RK. Chronic rhinosinusitis: epidemiology and cost. Allergy Asthma Proc 2013; 34(4):328-334.

(12) Smith W, Murphy C. Epidemiological Studies of Smell: Discussion and Perspectives. Annals of the New York Academy of Sciences, Volume 1170, Number 1, July 2009, pp. 569-573(5)

(13) Seemungal TA, Donaldson GC, Bhowmik A et al. Time course and recovery of exacerbations in patients with chronic obstructive pulmonary disease. Am J Respir Crit Care Med 2000; 161(5):1608-1613.

(14) Hurst JR, Vestbo J, Anzueto A et al. Susceptibility to exacerbation in chronic obstructive pulmonary disease. N Engl J Med 2010; 363(12):1128-1138.

(15) Quint JK, Donaldson GC, Goldring JJ et al. Serum IP-10 as a biomarker of human rhinovirus infection at exacerbation of COPD. Chest 2010; 137(4):812-822.

(16) Vachier I, Vignola AM, Chiappara G et al. Inflammatory features of nasal mucosa in smokers with and without COPD. Thorax 2004; 59(4):303-307. 
(17) Noguera A, Gomez C, Faner R et al. An investigation of the resolution of inflammation (catabasis) in COPD. Respir Res 2012; 13:101.

(18) Mallia P, Message SD, Kebadze T et al. An experimental model of rhinovirus induced chronic obstructive pulmonary disease exacerbations: a pilot study. Respir Res 2006; 7:116.

(19) Wilkinson TM, Hurst JR, Perera WR et al. Effect of interactions between lower airway bacterial and rhinoviral infection in exacerbations of COPD. Chest 2006; 129(2):317-324.

(20) Fark T, Hummel T. Olfactory disorders: distribution according to age and gender in 3,400 patients. Eur Arch Otorhinolaryngol 2013; 270(2):777-779.

(21) Jones PW, Harding G, Berry P et al. Development and first validation of the COPD Assessment Test. Eur Respir J 2009; 34(3):648-654.

(22) Mackay AJ, Donaldson GC, Patel AR et al. Usefulness of the Chronic Obstructive Pulmonary Disease Assessment Test to evaluate severity of COPD exacerbations. Am J Respir Crit Care Med 2012; 185(11):1218-1224.

(23) Miravitlles M, Garcia-Sidro P, Fernandez-Nistal A et al. Course of COPD assessment test (CAT) and clinical COPD questionnaire (CCQ) scores during recovery from exacerbations of chronic obstructive pulmonary disease. Health Qual Life Outcomes 2013; 11(1):147.

(24) Jones PW, Brusselle G, Dal Negro RW et al. Properties of the COPD assessment test in a cross-sectional European study. Eur Respir J 2011; 38(1):29-35.

(25) Dietz de Loos DA, Hopkins C, Fokkens WJ. Symptoms in chronic rhinosinusitis with and without nasal polyps. Laryngoscope 2013; 123(1):57-63.

(26) http://www.ginasthma.org/documents/1/Pocket-Guide-for-Asthma-Management-andPrevention. Update May 2014.

(27) Cabello H, Torres A, Celis R et al. Bacterial colonization of distal airways in healthy subjects and chronic lung disease: a bronchoscopic study. Eur Respir J 1997; 10(5):11371144.

(28) Miravitlles M, Marin A, Monso E et al. Colour of sputum is a marker for bacterial colonisation in chronic obstructive pulmonary disease. Respir Res 2010; 11:58.

(29) Soler N, Esperatti M, Ewig S et al. Sputum purulence-guided antibiotic use in hospitalised patients with exacerbations of COPD. Eur Respir J 2012; 40(6):1344-1353.

(30) Suh JD, Cohen NA, Palmer JN. Biofilms in chronic rhinosinusitis. Curr Opin Otolaryngol Head Neck Surg 2010; 18(1):27-31.

(31) Callebaut I, Hox V, Bobic S et al. Effect of nasal anti-inflammatory treatment in chronic obstructive pulmonary disease.Am J Rhinol Allergy. 2013 Jul-Aug;27(4):273-7. doi:

10.2500/ajra.2013.27.3887. 


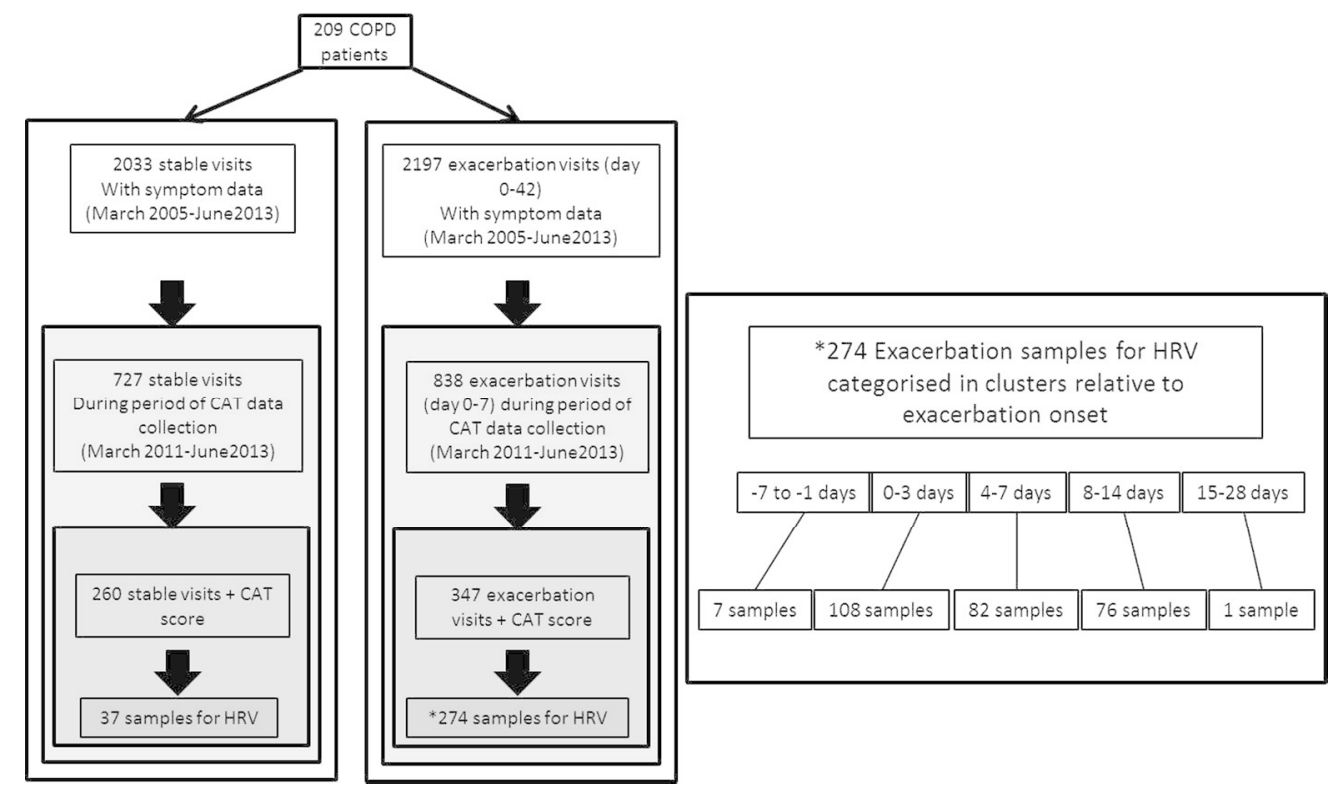

Figure 1. Flowchart of COPD patients included, data collected and samples processed during 8-years (2005$2013)$ in the London COPD cohort.

$240 \times 142 \mathrm{~mm}(150 \times 150 \mathrm{DPI})$ 


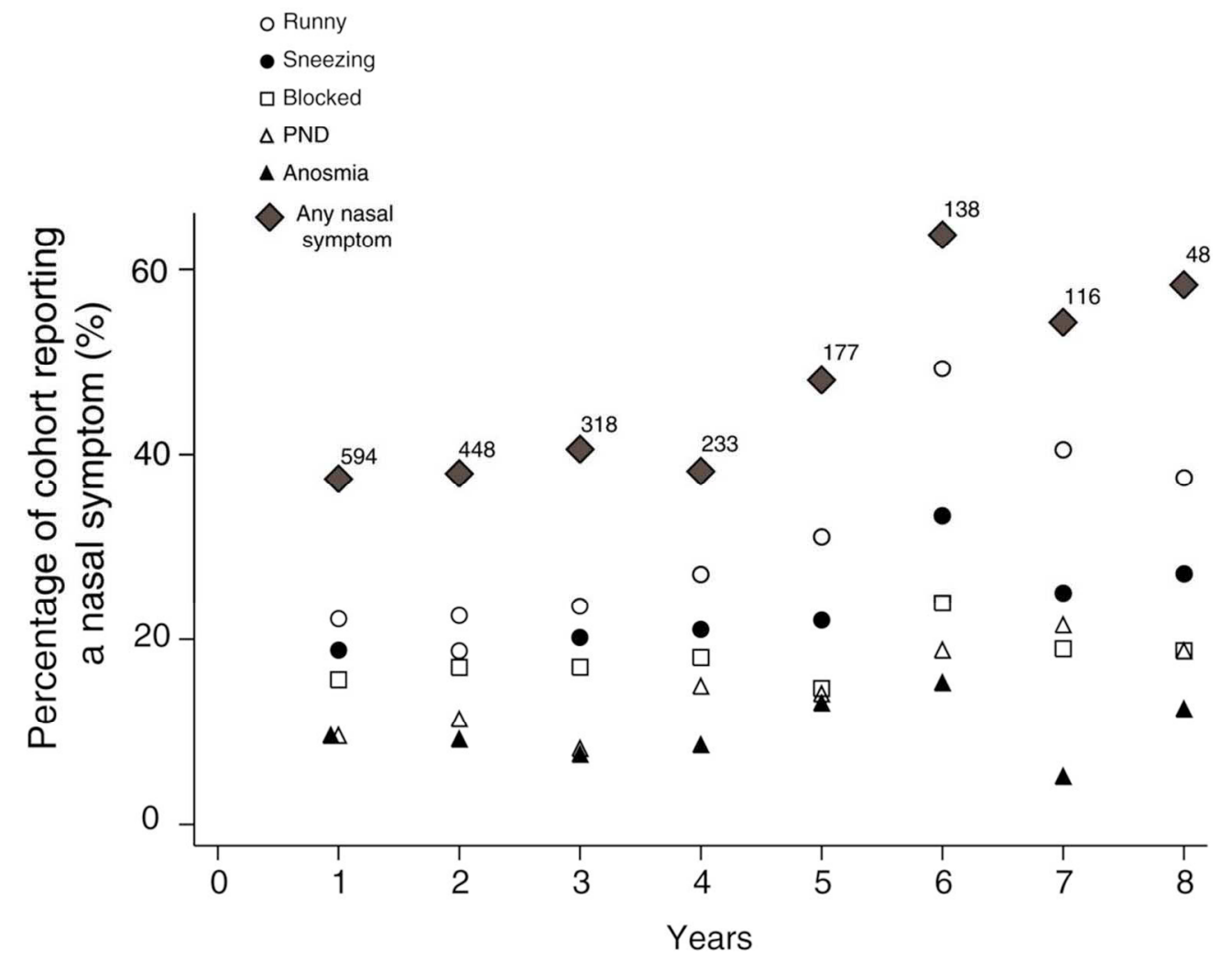

Figure 2. Percentage of patient-reported symptoms over time in the London COPD Cohort. Number refers to the number of visits where patients reported having nasal symptoms $249 \times 182 \mathrm{~mm}$ (134 x 146 DPI) 

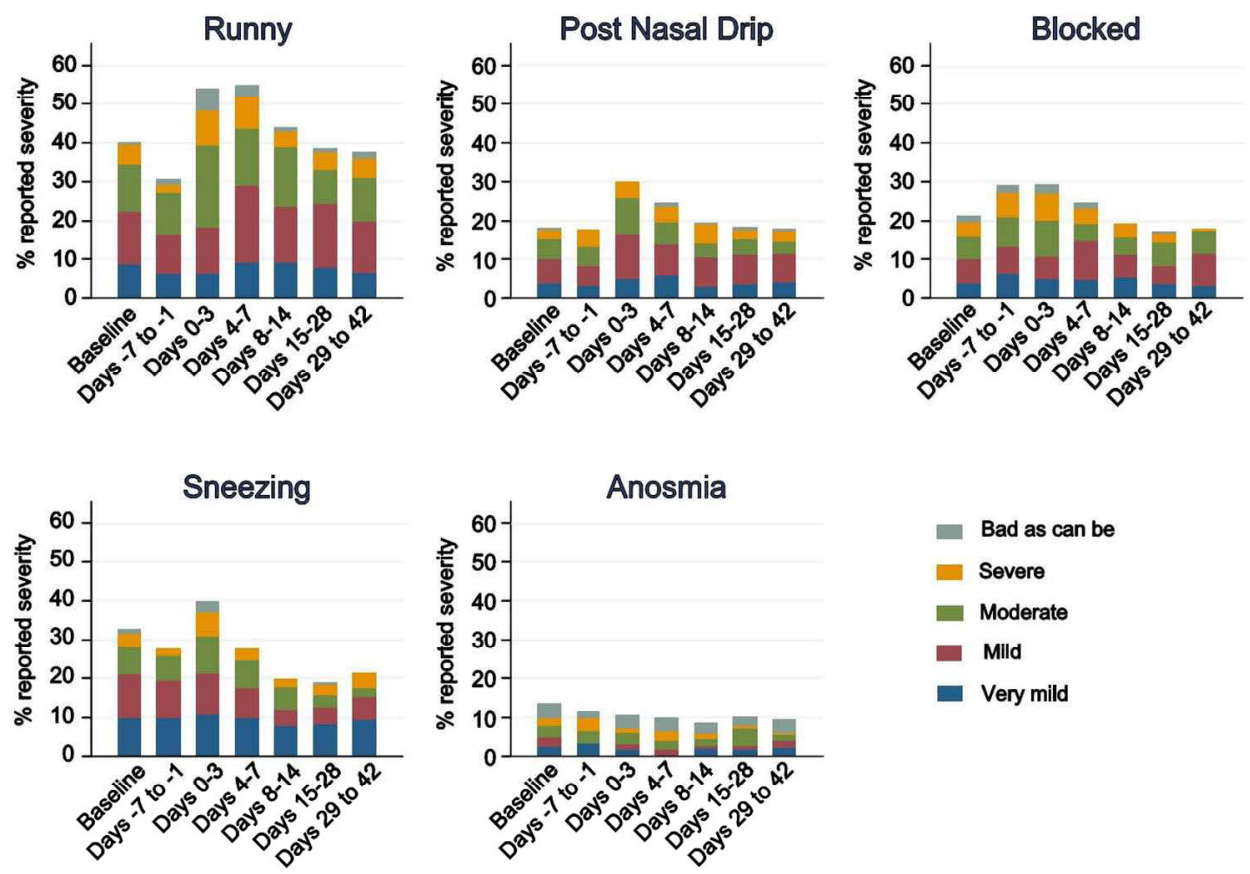

Figure. 3 Time course of nasal symptoms during exacerbations in the London COPD Cohort graded and divided by clusters of days. $254 \times 187 \mathrm{~mm}(150 \times 150 \mathrm{DPI})$ 

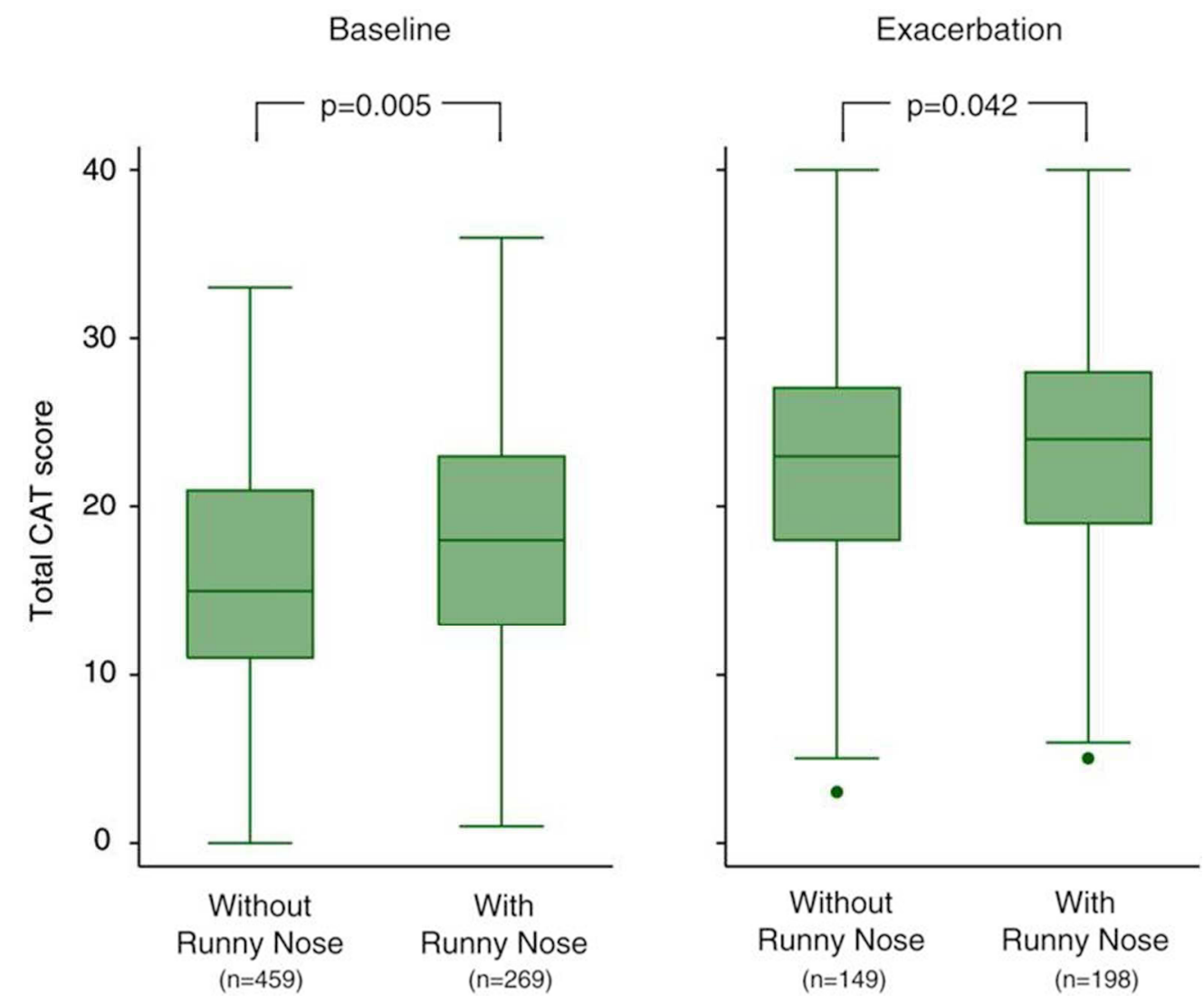

Figure 4. Effect of nasal discharge during stable state and at exacerbation and its impact on the CAT score. $135 \times 113 \mathrm{~mm}(150 \times 150 \mathrm{DPI})$ 

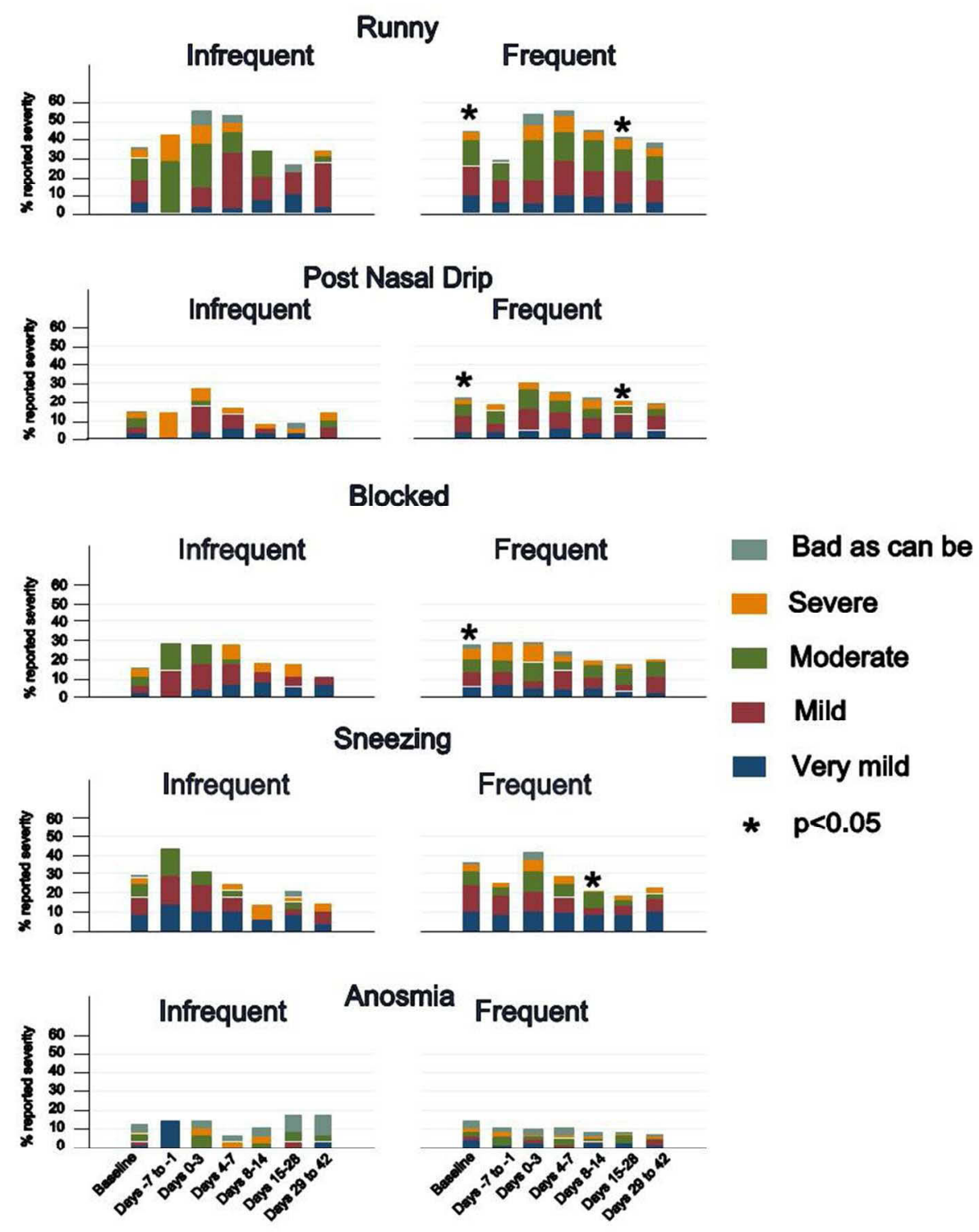

Figure. 5 Time course of nasal symptoms during exacerbations in patients with infrequent and frequent exacerbations. $* \mathrm{p}<0.05$, chi-squared test. $157 \times 190 \mathrm{~mm}(150 \times 150 \mathrm{DPI})$ 